\title{
HIGH-RESOLUTION MRI IN THE INVESTIGATION OF RECURRENT PAIN AFTER LUMBAR DISCECTOMY
}

\author{
SIMON CAVANAGH. JOHN STEVENS, JONATHON R. JOHNSON
}

From St Mary's Hospital, London, England

We used gadolinium-enhanced fat-suppressed MRI to investigate 67 patients with persistent pain after lumbar discectomy. Twenty-five patients had reoperations for lesions diagnosed in this way. Eleven were for recurrent disc prolapse at the same level and sciatica was relieved by all but one. Five operations were for prolapse at an adjacent level and all were successful.

The diagnosis of sepsis was less precise, but extension of tissue enhancement into the operated disc space was found to be significant. Only three patients had evidence of arachnoiditis which suggests that this condition has been too often diagnosed as a cause of persisting low back pain.

J Bone Joint Surg [Br] 1993; 75-B:524-8.

Received 25 August 1992; Accepted 22 October 1992

After lumbar discectomy, low back pain and sciatica are reported to recur in $5 \%$ to $10 \%$ of patients (Davis 1982). This may be related to lateral or central spinal stenosis or to arachnoiditis, but is usually due to residual or recurrent disc herniation or to epidural fibrosis.

The distinction between disc herniation and epidural fibrosis is important because reoperation is unlikely to succeed if scar tissue only is present. An epidural mass can be demonstrated by myelography, or better by CT or MRI but these methods cannot reliably establish its nature. This is because degenerate fibrocartilage and scar tissue have similar appearances on both CT and MRI; differentiation must depend on shape, position and the effects of the mass on the theca and nerve roots (Meyer et al 1982; Hochhauser et al 1988). Scar tissue is usually homogeneously vascularised, and degenerate fibrocartilage is avascular or vascularised only around its periphery; the use of an intravenous contrast medium to enhance vascularised tissues should therefore help to distinguish between them.

S. Cavanagh, MA, FRCS, Senior Registrar

J. Stevens, FRCR, Consultant Neuroradiologist

J. R. Johnson, FRCS, Consultant Orthopaedic Surgeon

St Mary's Hospital, Praed Street, London W2 INY, UK

Correspondence should be sent to $\mathrm{Mr} \mathrm{S}$. Cavanagh at the Royal National Orthopaedic Hospital Trust, Brockley Hill, Stanmore, Middlesex HA7 4LP, UK

(C) 1993 British Editorial Society of Bone and Joint Surgery $0301-620 \mathrm{X} / 93 / 4573 \$ 2.00$
The technique is of limited value in CT because the beam-hardening effect caused by the enclosing ring of dense bone reduces contrast discrimination within the spinal canal (Braun et al 1985; Firooznia et al 1987). This limitation does not apply to MRI, but the enhancement of an epidural mass may raise the signal from it to that of the surrounding epidural fat, thus tending to make such a mass inconspicuous or invisible. Many MRI units, however, can suppress the signal from epidural fat so that the enhancement of vascularised tissue and epidural veins is made clear. Lack of definite enhancement of the whole mass is then predictive of residual or recurrent disc material. Modern MRI units can also resolve intradural roots, by using appropriate pulse sequences, and therefore should be capable of demonstrating reliably the intradural adhesions which are the hallmark of arachnoiditis (Stevens and McAllister 1991).

We have tested the reliability of fat-suppressed, contrast-enhanced MRI comparing preoperative images thought to show residual disc material with the findings at operation. We also obtained data on the prevalence of intradural adhesions after routine lumbar discectomy.

\section{PATIENTS AND METHODS}

We performed MRI on 67 patients (41 men and 26 women) whose average age was 46 years 2 months ( 18 to 75). All had undergone previous lumbar disc surgery or injection, and had recurrent low back pain, symptoms of radiculopathy, and some functional disability. Three of these patients had had only a chemical discectomy with chymopapain.

For MRI we used a 1.5 Tesla superconducting unit (Signa, General Electric, Slough, UK), making four separate data acquisitions in each case. The acquisition matrices were $256 \times 192$ using $4 \mathrm{~mm}$ slice thickness and a $2 \mathrm{~mm}$ interslice gap. Sagittal sequences were both T1weighted (TR $500 \mathrm{~ms}$, TE $10 \mathrm{~ms}$ ), spin-density weighted and T2-weighted (TR $2000 \mathrm{~ms}$, TE $80 \mathrm{~ms}$ ). Axial T1weighted (TR $600 \mathrm{~ms}$, TE $13 \mathrm{~ms}$ ) acquisitions were obtained angled to the relevant disc space before and after the intravenous injection of 10 to $15 \mathrm{ml}$ of gadolinium diethylenetriaminepenta-acetic acid (DPTA). To maximise the delineation of enhanced scar tissue we used a modified fat-suppression pulse sequence. We also used a multiplanar gradient rephasing (MPGR) 

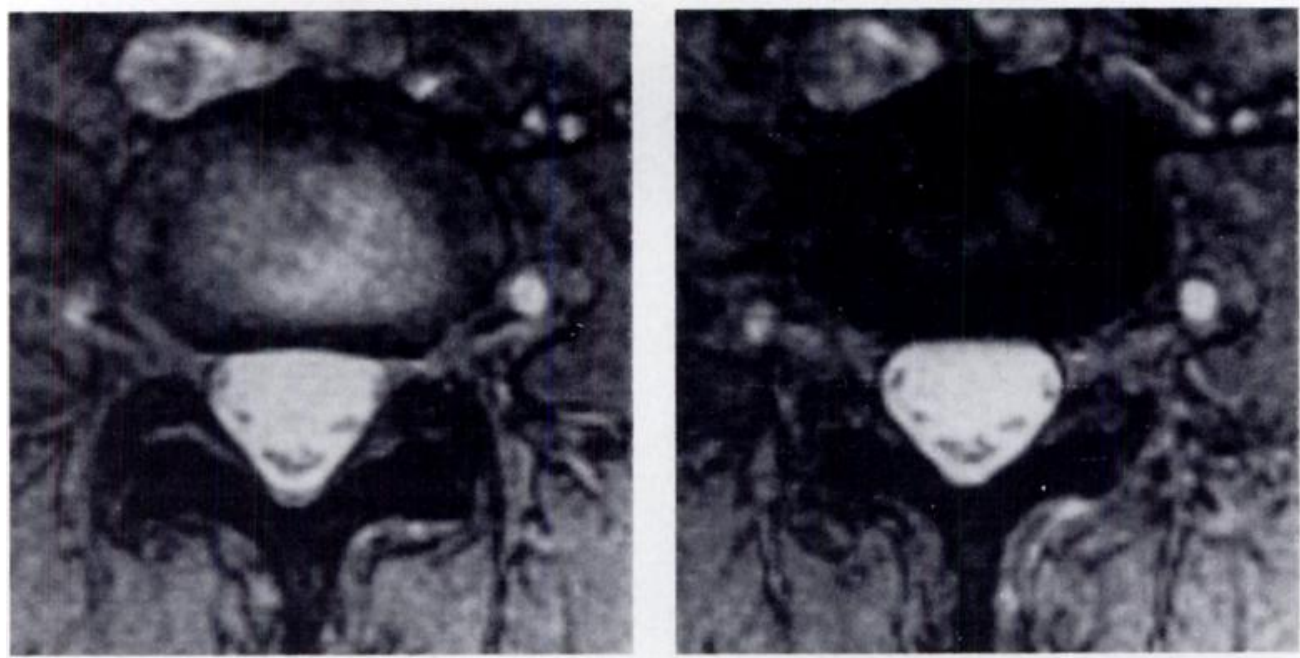

Fig. 1

A multiplanar gradient rephasing sequence which enhances the intradural CSF and shows the intradural adhesions between the nerve roots and the theca, characteristic of arachnoiditis.
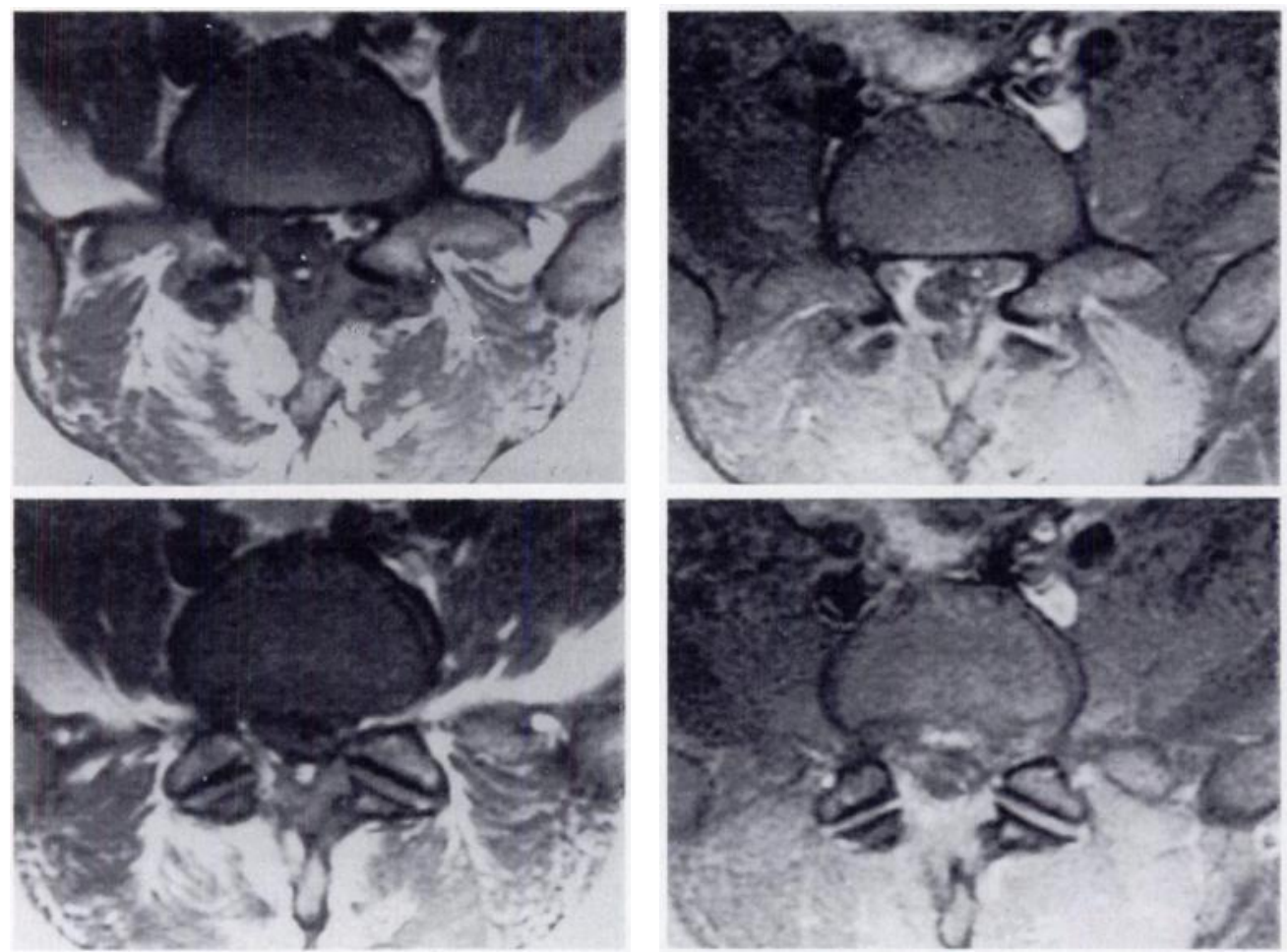

Fig. 2a

Fig. $2 b$

Epidural scarring. Figure 2a - The precontrast MR scan shows a right-sided epidural mass which suggests recurrent disc prolapse. Figure $2 b$ - After contrast, with fat-suppressed imaging, the mass demonstrates the uniform enhancement characteristic of epidural scarring.

sequence which highlighted the cerebrospinal fluid and facilitated the identification of arachnoiditis (Fig. 1).

\section{RESULTS}

The scans were examined by a radiologist (JS) with a special interest in the central nervous system and spine. The radiological diagnosis in all 67 patients is shown in Table I. One scan showed some disc bulging but no prolapse, and two were reported as normal. There was evidence of arachnoiditis on only three scans; one of these also had a recurrent disc lesion.

In 29 patients the radiological diagnosis was epidural scarring (Fig. 2) and no further operation was performed. No procedure was undertaken in another four with degenerative changes only and no suggestion of nerveroot entrapment.

Surgery was performed in 25 of the 29 patients in whom the scan indicated disc herniation, infection or spinal stenosis. The operations were carried out by one 
of six surgeons, three orthopaedic surgeons and three neurosurgeons.

Of the 14 patients in whom the scan had shown a recurrent disc prolapse at the same level, 11 had reoperations. In this group the average time since the first operation was 53 months (6 to 190), and the radiological diagnosis was confirmed in all cases (Fig. 3). The operation gave substantial early relief from sciatic pain in all patients and, at a minimum follow-up of 12 months, only one has persistent sciatic pain although three have occasional low back pain. Scans were repeated at six months; they showed scarring around the nerve root with mild narrowing of the thecal sac.

Three patients with scans indicating recurrent disc prolapse did not have reoperations. One prolapse was contralateral to the symptomatic side, which showed only scarring around the nerve root, and the other two

Table I. Radiological diagnoses and number of reoperations in 67 patients with persistent pain after lumbar discectomy

\begin{tabular}{lll}
\hline Diagnosis & Number & Reoperation \\
\hline Epidural scarring & 29 & 0 \\
Recurrent disc herniation & $14^{*}$ & 11 \\
Infection & 5 & 4 \\
Herniated disc at another level & 5 & 5 \\
Central or lateral stenosis & 5 & 5 \\
Degenerative changes & 4 & 0 \\
Arachnoiditis & $3^{*}$ & 0 \\
No pathology & 3 & 0 \\
\hline
\end{tabular}

* one patient had both arachnoiditis and a recurrent disc herniation

patients declined further surgery. Both have improved slightly but remain symptomatic.

Five patients showed disc prolapse at levels adjacent to the original one; all gained relief from reoperation. Five patients showed central or lateral canal stenosis: three gained substantial relief from reoperation and two had some amelioration.

Five patients had radiological appearances indicating infection: four had reoperation and the other was treated conservatively. In this case the scan had indicated infection at L4/L5 and L5/S1 with extensive enhancing intraspinal granulation tissue. The patient was diabetic and on steroids; he responded to treatment with antibiotics and a brace and obtained spontaneous fusion after four months. In one of the four who had a reoperation the scan showed an extraspinal abscess surrounded by enhanced granulations tracking into the L4/L5 disc space (Fig. 4). The abscess cavity was evacuated; pus cells were found but no organisms were isolated. In a second patient a retroperitoneal exploration confirmed chronic discitis at L2/L3. Evacuation of the infected material and a course of antibiotics relieved the symptoms. In the other two patients the scans failed to correlate with the operative findings. In one there was a meningocele with a large dural tear and decompression of the tense cyst relieved symptoms. In the other patients the scan suggested an abscess seven years after chemonucleolysis. No pus was found at operation, and the MRI appearance of an extraspinal fluid collection remained unexplained.

\section{DISCUSSION}

Gadolinium-DPTA is a paramagnetic agent which alters the relaxation properties of the hydrogen atoms in surrounding tissue water. At the concentrations used in clinical practice, shortening of the spin-lattice (T1) relaxation time predominates, so that in Tl-weighted images (short TR/TE images) the result is an increased signal. Fat also yields a high signal on such images, and demonstration of enhancement is therefore greatly facilitated by suppressing the fat signal. This is achieved by applying a prior radiofrequency pulse of the same resonance frequency as fat. This frequency is slightly different from that of the protons in water, and eliminates the fat signal from the imaging pulse sequence.

We did not explore patients in whom uniform enhancement of extradural tissue was seen, and have no confirmation therefore of the diagnosis of scarring. All 14 patients, however, who consented to reoperation after imaging had indicated recurrent disc prolapse had their diagnoses confirmed surgically.

The imaging diagnosis of postoperative infection was less satisfactory. In five patients enhanced MRI showed a fluid collection in the extraspinal tissues surrounded by an enhanced wall of variable thickness and definition. In two, paraspinal abscesses were confirmed; in these and in the patient treated conservatively, the enhancement extended into the previously operated disc. In the two patients with no abscess at operation, the lesion did not enter the disc space. All such postoperative collections do not represent infection: some may be pseudomeningoceles, others the result of organised haematomas or tissue necrosis.

Our study has shown that enhancement in scar tissue persists for many years. In six cases the first operation was five years earlier, and in one, nine years. All seven patients showed conspicuous enhancement of the operative track close to and within the laminectomy as well as in the spinal canal. It has been reported that scar tissue enhances maximally at about three months, then decreasingly, possibly becoming undetectable after two years (Ross et al 1989). It seems that the increased sensitivity of more modern equipment, and the use of fat suppression, have overcome this limitation. It has also been reported that this type of MRI, within three months of operation, often shows enhanced and non-enhanced epidural masses, and apparent enhancement in the decompressed root in patients with good or excellent 
results (Stevens and McAllister 1991). Confusing appearances have also been seen on early postoperative CT.

It is clear that caution should be exercised before ascribing clinical features to changes shown by postoperative imaging, especially during the first three months. Tissue reactions may not have settled and nonvascularised areas of haematoma and tissue necrosis may simulate residual disc material or abscesses. In our series all but one case had an interval of at least six months between operation and postoperative imaging.

We found only three cases of arachnoiditis, and all these had had previous myelography using Myodil (Glaxo, Uxbridge, UK). This suggests that arachnoiditis due to lumbar disc surgery has probably been overdiagnosed in the past.

Complex and expensive imaging is of little value
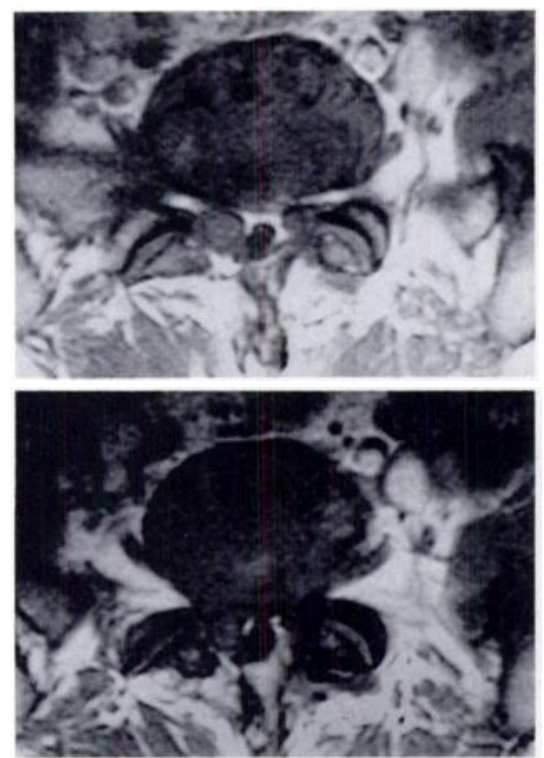

Fig. 3a
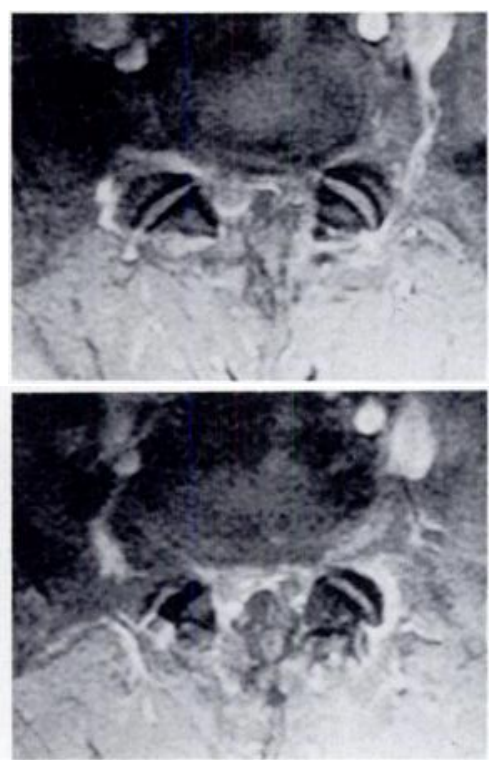

Fig. 3b

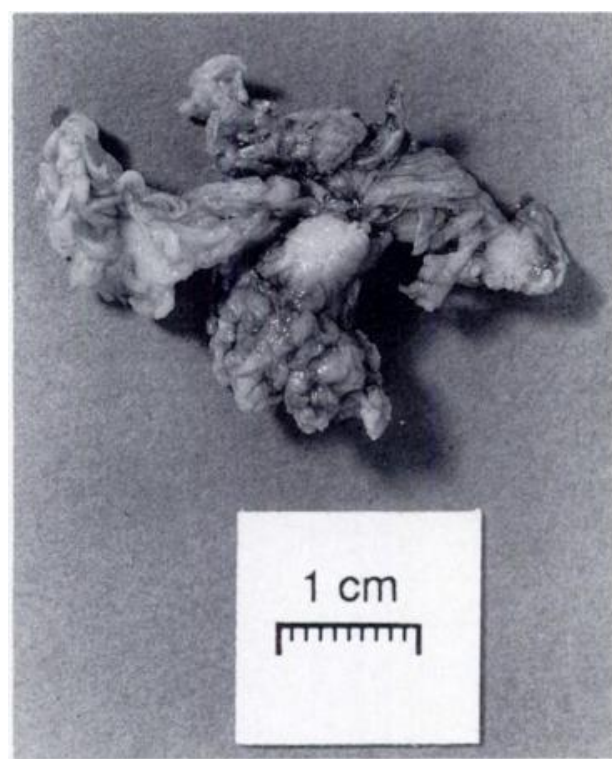

Fig. 3c

Recurrent disc lesion. Figure 3a - The precontrast MR scan shows a large right-sided lesion suggestive of disc material. Figure $3 \mathrm{~b}-\mathrm{The}$ postcontrast image confirms that disc material, with an attenuated intensity, is prolapsing into the epidural space. There is an enhancing scar posteriorly. Figure 3c-Recurrent disc fragment excised at operation.

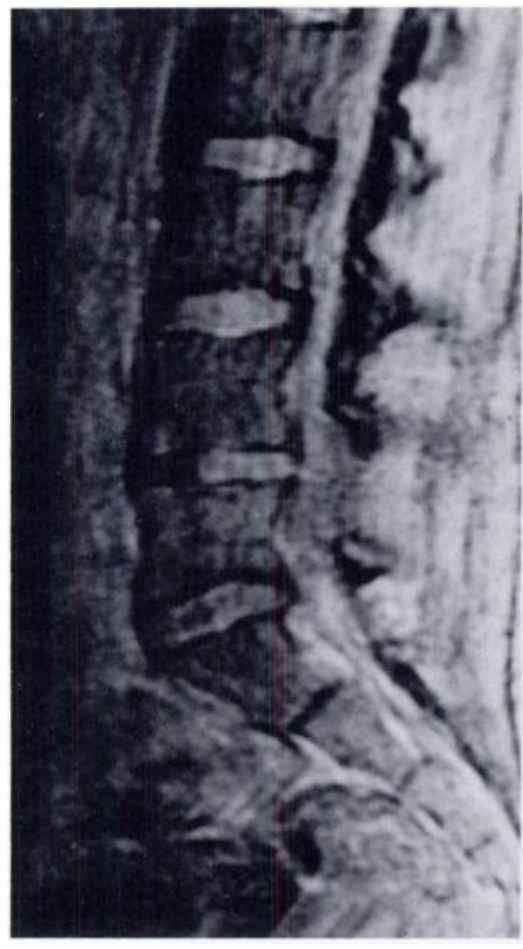

Fig. 4a

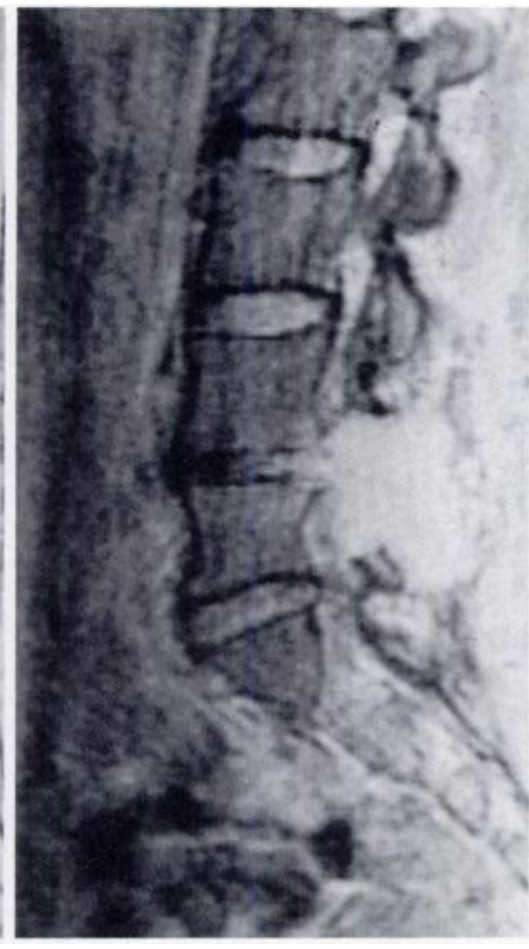

An extraspinal abscess, showing enhancing granulations tracking into the L4/L5 disc space. Figure $4 \mathrm{a}-$ Sagittal views. Figure $4 \mathrm{~b}-$ Axial views.
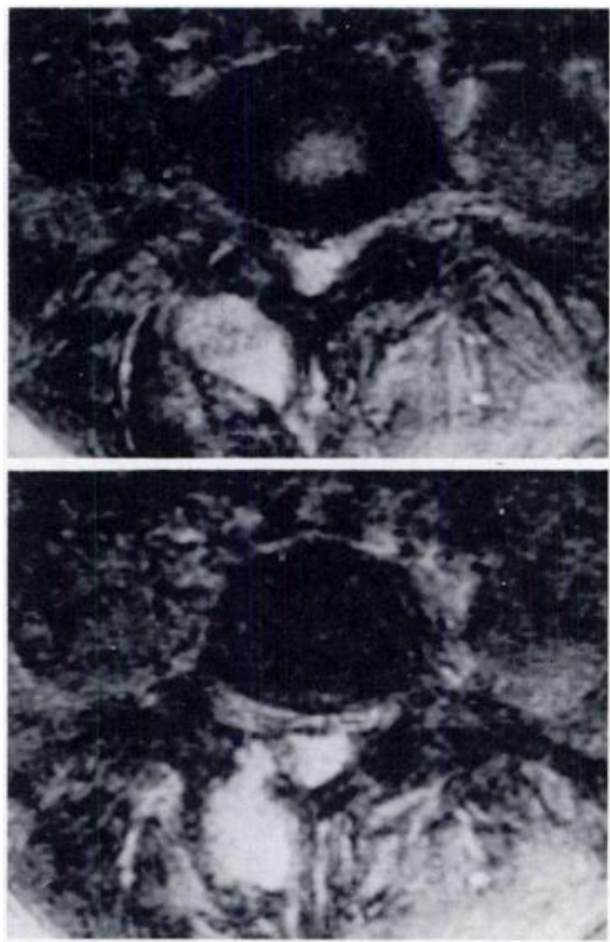

Fig. 4b 
unless the findings lead to surgery which provides symptomatic relief. The good relief in our 14 patients with recurrent disc prolapse confirms our view that the technique is valuable in the assessment of recurrent low back pain and sciatica after surgery.

The authors are grateful to Alan Crockard FRCS, Michael Powell FRCS, and Richard Hayward FRCS at the National Hospital for Nervous Diseases, Queen Square, London and also to Andrew Ransford FRCS and Michael Sullivan FRCS at the Royal National Orthopaedic Hospital Trust, Stanmore, Middlesex for their help in reporting their cases.

No benefits in any form have been received or will be received from a commercial party related directly or indirectly to the subject of this article.

\section{REFERENCES}

Braun IF, Hofiman JC, Davis PC, Landman JA, Tindall GT. Contrast enhancement in CT differentiation between recurrent disk herniation and postoperative scar. $A J R$ 1985; $145: 785-90$
Davis CH, Jr. Extradural spinal cord and nerve root compression from benign lesions of the lumbar area. In: Youmans JR, ed. Neurological surgery. Second ed, Vol. 4. Philadelphia, etc: W. B. Saunders Co, 1982:2535-61.

Firooznia H, Kricheff II, Rafi M, Golimbu C. Lumbar spine after surgery: examination with intravenous contrast-enhanced CT. Radiology 1987; 163:221-6.

Hochhauser L, Kiefier SA, Cacayorin ED, Petro GR, Teller WF. Recurrent postdiskectomy low back pain: MR - surgical correlation. AJR 1988: $151: 755-60$.

Meyer JD, Latchaw RE, Roppolo HM, Ghoshhajra K, Deeb ZL. Computed tomography and myelography of the postoperative lumbar spine. AJNR 1982; 3:223-8.

Ross JS, Blaser S, Masaryk TJ, et al. Gd-DPTA enhancement of posterior epidural scar: an experimental model. AJNR 1989; 10:1083-8

Stevens JM, McAllister VL. The imaging of spinal pathology. In: Grainger RG, Allison DJ, ed. Diagnostic radiology: an AngloAmerican textbook of imaging. Second ed. Edinburgh, etc: Churchill Livingstone, $1991: 2045-74$ 